Honam Mathematical J. 32 (2010), No. 1, pp. 53-60

\title{
DISCRETE CURVATURE BASED ON AREA
}

\author{
KYEONGSU PARK
}

\begin{abstract}
The concept of discrete curvature is a discretization of the curvature. Many literatures introduce discrete curvatures derived from arc length of circular arcs. We propose a new concept of discrete curvature of a polygon at each vertex, which is derived from area of fan shapes. We estimate the error of the discrete curvature and compare the discrete curvature with old one.
\end{abstract}

\section{Introduction}

The curvature of a smooth curve at a point is determined by the sharpness of the turning. This concept does not depend on the isometric embedding or the regular reparamerization but only on the shape of the curve. This intrinsic concept can be applied to a polygon and we call it the discrete curvature. Since the discrete curvature is determined by the shape of a polygon as a smooth curve, it is applied to discrete geometry and computer vision.

Many literatures introduce discrete curvatures in different ways and derive applications([1], [2], [3], [4], [6]). A formula of the discrete curvature, which will be denoted by $\bar{k}$ in this paper, is originated from the arc length equation of a circle. The circular arc with radius $r$ and central angle $\theta$ is of length $l=r \theta$. Therefore the curvature of the circular arc is $1 / r=\theta / l$. This equation is the motivation of the discrete curvature based on arc length.

In this paper we introduce a discrete curvature of a polygon at each vertex, which is derived from the area equation of a fan shape. A fan shape with radius $r$ and central angle $\theta$ has its area $s=r^{2} \theta / 2$. Hence the curvature is given by $1 / r=\sqrt{\theta /(2 s)}$. Using this equation we will define a discrete curvature $k$, which is called the discrete curvature based on area.

Received January 26, 2010. Accepted February 23, 2010.

2000 AMS Mathematics Subject Classification: 68U05

Key words and phrases: discrete curvature 
Our new discrete curvature $k$ is closer than $\bar{k}$ to the curvature of the smooth curve in case of $y=x^{2}$. But $\bar{k}$ is closer than $k$ in case of a circle.

In section 2 , we define the discrete curvature based on area and compute the error in contrast to the curvature of a smooth curve. In section 3 , we compare three discrete curvatures $1 / r, k$ and $\bar{k}$ numerically.

\section{A discrete curvatures}

The circular arc of curvature $\kappa$ is of radius $1 / \kappa$. The arc length $l$ of a circular arc with central angle $\theta$ is $l=\theta / \kappa$. Hence the curvature is given by the equation $\kappa=\theta / l$. We introduce a discrete version of the curvature originated from this equation, see [2]. Let $\{A, P, B\}$ be a polygon. We define $\bar{k}_{p}$, or $\bar{k}$ simply, at vertex $P$ by

$$
\bar{k}_{p}=\frac{2(\pi-\phi)}{l_{1}+l_{2}}
$$

where $\phi=\angle A P B, l_{1}=|A-P|$ and $l_{2}=|B-P| . \quad \bar{k}_{p}$ is called the discrete curvature based on arc length.

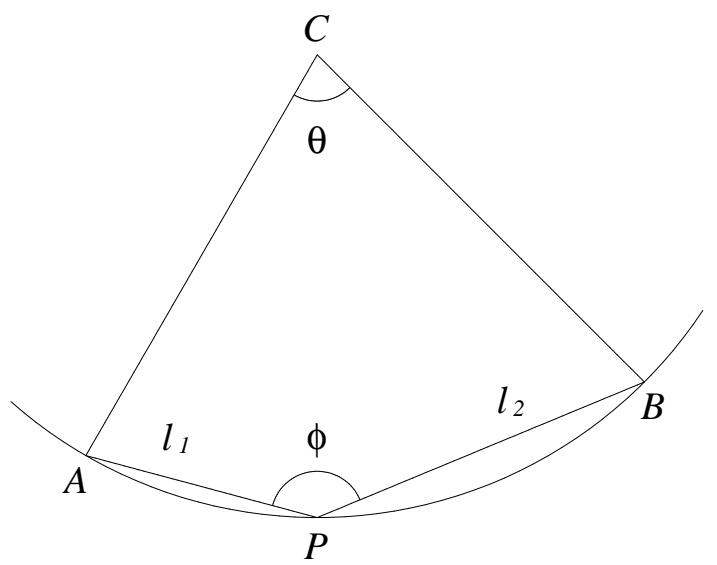

FiguRE 1. The circumscribed circle of the triangle $A P B$

To examine the validity of (1), let $C$ be the center of the circumscribed circle of the triangle $A P B$ as shown in the Figure 1 . Then $2(\pi-\phi)=\theta$ and $\bar{k}=\theta /\left(l_{1}+l_{2}\right)$. Since $l_{1}+l_{2}$ approximates the arc length of the circular arc from $A$ to $B$, so does $\bar{k}$ the curvature. Obviously $\bar{k} \rightarrow \kappa$ as $l_{1}+l_{2} \rightarrow 0$. 
One may find the smooth analogue of this situation in the book [5] and $[6]$.

In $[2]$, the authors estimates the error of $\bar{k}$.

Theorem 1. Let $\alpha$ be a regular $C^{\infty}$ curve and $\{A, P, B\}$ a polygon on $\alpha$. Then $\bar{k}$ satisfies:

$$
\bar{k}=\kappa+O\left(l_{1}+l_{2}\right) .
$$

The area $s$ of the fan shape with angle $\theta$ bounded by the circular arc of curvature $\kappa$ and two radii is $s=\theta /\left(2 \kappa^{2}\right)$. From this equation we get the curvature of a circular arc as following:

$$
\kappa=\sqrt{\frac{\theta}{2 s}} .
$$

Our definition of the discrete curvature is motivated from this equation.

Definition 1. Given a polygon $\{A, P, B\}$, let $C$ be the intersection of two perpendicular bisectors of line segments $A P$ and $P B$ as shown in the Figure 2. The discrete curvature $k_{p}$, or $k$ simply, of the polygon is defined by

$$
k_{p}=\sqrt{\frac{\theta}{2\left(s_{1}+s_{2}\right)}}
$$

where $s_{1}, s_{2}$ are the areas of the triangles $C A P, C B P$, resp. and $\theta$ is the angle $\angle A C B$. We call $k_{p}$ the discrete curvature based on area.

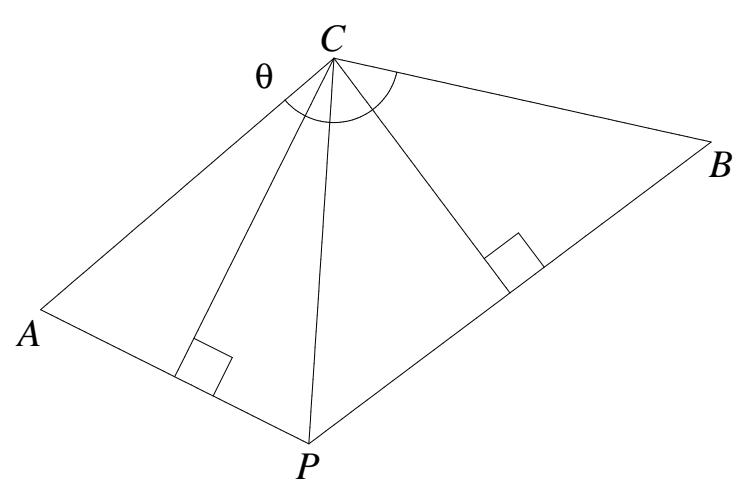

FIGURE 2. Discrete curvature of a polygon 
For a sufficiently small polygon inscribed in a circle the equation (2) approximates the curvature with small error. To approximate error in the smooth case, suppose that $\alpha=\left(\alpha_{1}, \alpha_{2}\right)$ is a regular $C^{\infty}$ curve. Let $\{A, P, B\}$ be points on the curve $\alpha$ given by $A=\alpha(a), P=\alpha(0)$ and $B=\alpha(b)$ with $a<0<b$. By compositions of a translation and rotation we may assume that

$$
\alpha(t)=\left(t, \frac{1}{2} \kappa t^{2}+R_{2}(t)\right)
$$

where $\kappa$ is the curvature of $\alpha$ at $t=0$ and $R_{2}$ the remainder of the Taylor polynomial of $\alpha_{2}$ at 0 .

For any $t \in[a, b]$ the remainder $R_{2}(t)$ satisfies

$$
\begin{aligned}
\left|R_{2}(t)\right| & =\left|\int_{0}^{t} \alpha_{2}^{(3)}(u) \frac{u^{2}}{2 !} d u\right| \\
& \leq \max \left\{\left|\alpha_{2}^{(3)}(u)\right| \mid a \leq u \leq b\right\} \frac{|t|^{3}}{6} .
\end{aligned}
$$

Hence $R_{2}(t)=R(t) t^{3}$ for some function $R$, which is bounded by a constant on the interval $[a, b]$.

Lemma 1. The radius $r$ of the circumscribed circle of the triangle $A P B$ satisfies

$$
1 / r=\kappa+O(\lambda)
$$

where $\lambda=\max \{|a|,|b|\}$.

Proof. . Let $C$ be the point in Definition 1. The circumscribed circle of the triangle $A P B$ has its center at $C$ and radius $|C-P|$. Using the equation (3) we can estimate $C$ and get

$$
C=\left(O(\lambda), \frac{1}{\kappa}+O(\lambda)\right) .
$$

Therefore the radius satisfies $r=1 / \kappa+O(\lambda)$ and we get the desired result.

The above Lemma implies that the radius $r$ is a candidate of the discrete curvature.

Now we estimate the discrete curvature $k$.

Theorem 2. The discrete curvature $k$ of the polygon $\{A, P, B\}$ as above satisfies

$$
k=\kappa+O(\lambda)
$$

where $\lambda=\max \{|a|,|b|\}$. 
Proof. . Let $\theta_{1}=\angle A C P$ and $\theta_{2}=\angle B C P$. Then the area of the triangle $C A P$ is $s_{1}=r^{2} \sin \theta_{1} / 2$ and that of $C B P$ is $s_{2}=r^{2} \sin \theta_{2} / 2$. The discrete curvature $k$ of the polygon $\{A, P, B\}$ at $P$ is given by

$$
k=\sqrt{\frac{\theta_{1}+\theta_{2}}{2\left(s_{1}+s_{2}\right)}}=\frac{1}{r} \sqrt{\frac{\theta_{1}+\theta_{2}}{\sin \theta_{1}+\sin \theta_{2}}}
$$

By the Lemma $1 / r=\kappa+O(\lambda)$. The second term of (4) can be estimated using the property of equilateral triangle and $\sin \theta=\theta+O\left(\theta^{3}\right)$.

Corollary 1. $k$ tends to $\kappa$ as $A$ and $B$ tends to $P$.

\section{Comparison}

Let $\left\{P_{1}, \ldots, P_{n}\right\}$ be a polygon on a curve $\alpha$. One may expect that discrete curvatures has near value to the curvature of $\alpha$ at each vertex. In general it is hard to expect that such situation will arise. But discrete curvatures can approximate the curvature with sufficiently small errors whenever $\left|P_{i}-P_{i+1}\right|$ is sufficiently small for all $i$, because three discrete curvatures converges to the curvature of $\alpha$ at $P_{i}$ when $\left|P_{i-1}-P_{i}\right|$ and $\left|P_{i+1}-P_{i}\right|$, or equivalently $\left|P_{i-1}-P_{i}\right|+\left|P_{i+1}-P_{i}\right|$, tends to 0 ,

Now we will compare three discrete curvatures $k, \bar{k}$ and $1 / r$ defined in the above section. For a curve $\alpha$ three points $A=\alpha(a), P=\alpha(0)$, $B=\alpha(b)$ with $a<0<b$ form a polygon. We will compute and compare three discrete curvatures of the polygon at $P$ for some $a$ and $b$.

First of all, consider the polygon $\{A(\cos a, \sin a), P(1,0), B(\cos b, \sin b)\}$ with $-\pi<a<0<b<\pi$ on the unit circle as the Figure 3 . Then we have

and

$$
\begin{gathered}
k_{p}=\sqrt{\frac{a+b}{\sin a+\sin b}}, \\
\bar{k}_{p}=\frac{a+b}{2(\sin (b / 2)-\sin (a / 2))}
\end{gathered}
$$

$$
\frac{1}{r}=1
$$

We display the variance of discrete curvatures in case of $b=-a$ and $b=-2 a$ in the Figure 4. since $r$ is the radius of the circumscribed circle of the triangle $A P B$, it is independent of $a$ and $b$ and $1 / r=\kappa=1 . \bar{k}$ is closer to the curvature 1 than $k$. 


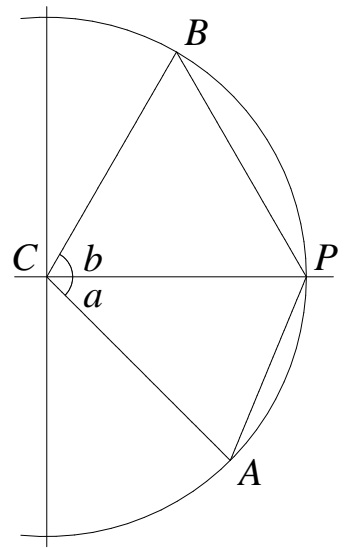

Figure 3. A polygon on the unit circle
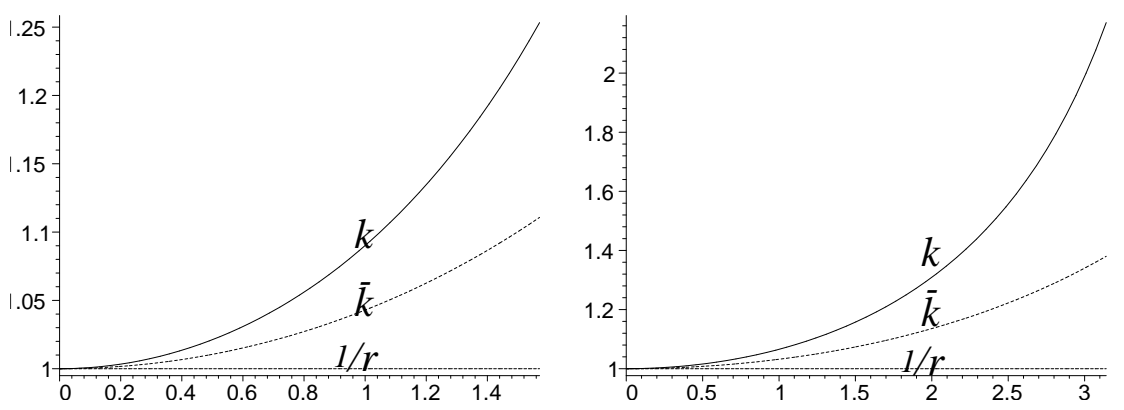

Figure 4. Curvatures of polygons on the unit circle. $x$ axis is the angle $b$ and $y$-axis is curvatures. In the left figure $b=-a$ and in the right one $b=-2 a$.

Now we consider the polygon on the graph of $y=x^{2}$ given by $\left\{A\left(a, a^{2}\right), P(0,0), B\left(b, b^{2}\right)\right\}$ with $a<0<b$ as the Figure 5. Then $r$ is given by

$$
r=\frac{\sqrt{\left(1+a^{2}\right)\left(1+b^{2}\right)\left(1+(a+b)^{2}\right)}}{2} .
$$

Using this equation we may compute $k$. The equation of $k$ and $\bar{k}$ is fairly complicated.

The Figure 6 represents the variance of discrete curvatures in case of $b=-a$ and $b=-2 a . k$ is closest to the curvature 2 of $y=x^{2}$ at $x=0$. Although the discrete curvature $1 / r$ is originated from the radius of the osculating circle, it is far from the curvature $\kappa$ in this case. 


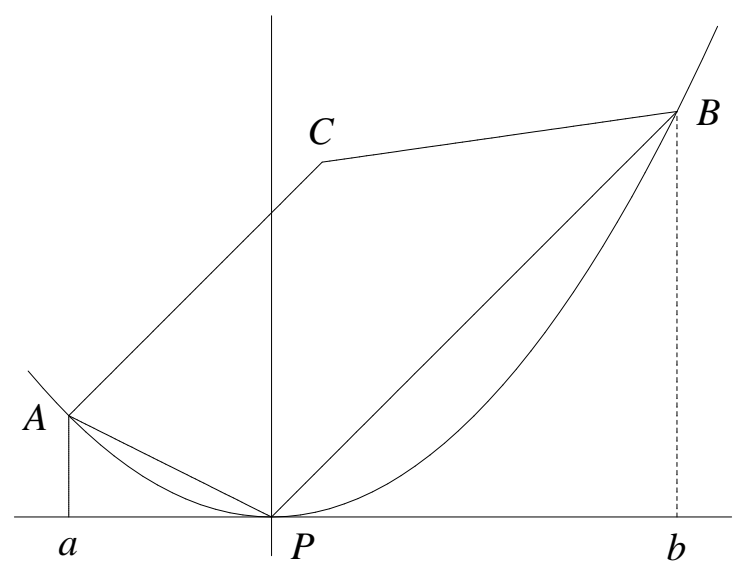

FiguRe 5. A polygon on the graph of $y=x^{2}$
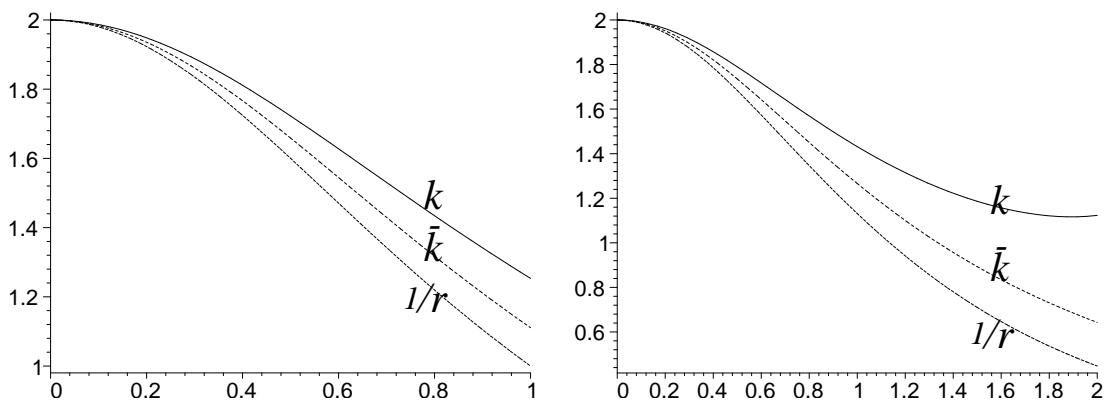

Figure 6. Curvatures of polygons on the graph of $y=$ $x^{2} . x$-axis is $b$ and $y$-axis is curvatures. In the left figure $b=-a$ and in the right one $b=-2 a$.

It is hard to estimate accruately an infinitesimal concept. Our new discrete curvature is closer to the curvature than the other in some cases, but not in another cases. Nevertheless this curvature can be applied to problems of edge detection, object recognition, pattern analysis and so on. 


\section{References}

[1] T. Adamek, O. Connor, A multiscale representation method for nonrigid shapes with a single closed contour, IEEE Transactions on Circuits and Systems for Video Technology, 14(2004), 742-753.

[2] V. Borrelli, F. Cazals, J-M. Morvan, On the angular defect of triangulations and the pointwise approximation of curvatures, Computer Aided Geometric Design 20(2003), 319-341.

[3] M. Jeon, D. Han, K. Park, G. Choi, Ternary univariate curvature-preserving subdivision, J. Appl. Math. \& Computing 18(2005), No. 1-2, 235-246

[4] Lewiner, T., Arc-length based curvature estimator. In SIBGRAPI'04(2004), 250257.

[5] M. M. Lipschutz, Theory and problems of differential geometry, McGraw-Hill Book Company, 1969.

[6] H. Liu, L. J. Latecki, W. Liu, A unified curvature definition for regular, polygonal, and digital planar curves, International Journal of Computer Vision 80(2008), 104-124.

Kyeongsu Park

Department of Game

Jeonju University

Jeonju 560-759, Korea

E-mail: pine@jj.ac.kr 